\title{
Editorial
}

\section{Function Spaces, Approximation Theory, and Their Applications}

\author{
Carlo Bardaro, ${ }^{1}$ Ioan Rasa, ${ }^{2}$ Rudolf L. Stens, ${ }^{3}$ and Gianluca Vinti ${ }^{1}$ \\ ${ }^{1}$ Department of Mathematics and Computer Sciences, University of Perugia, Via Vanvitelli 1, 06123 Perugia, Italy \\ ${ }^{2}$ Technical University of Cluj-Napoca, Cluj-Napoca, Romania \\ ${ }^{3}$ Lehrstuhl A für Mathematik, RWTH Aachen, Templergraben 55, 52056 Aachen, Germany \\ Correspondence should be addressed to Carlo Bardaro; carlo.bardaro@unipg.it
}

Received 8 August 2016; Accepted 9 August 2016

Copyright @ 2016 Carlo Bardaro et al. This is an open access article distributed under the Creative Commons Attribution License, which permits unrestricted use, distribution, and reproduction in any medium, provided the original work is properly cited.

The purpose of this special issue was to present new developments in the theory of function spaces, along with the deep interconnections with approximation theory and the applications in various fields of pure and applied mathematics. The reaction of the mathematical community was very satisfactory. We collected thirty-five submissions, covering a wide range of mathematical topics, ten of which were found to be suitable for publications in this issue. The major part of the accepted papers treats function spaces and their applications. In this respect, in the article by $\mathrm{X}$ Yang et al. a new class of function spaces, named "multi- $\beta$ normed spaces", is introduced, in connection with stability properties of certain type of functional equations, while, in the paper by A. A. Bakery, sequential spaces of Orlicz type are studied and connected with the theory of summability. In the review paper by L. Angeloni and G. Vinti, the approximation theory in the space of functions with bounded variation is developed, in view of applications to signal processing. Different notions of variation are considered and several approximation theorems for families of integral or discrete type operators are given. In the more theoretical article by $\mathrm{S}$. Wulede et al., a new class of Banach spaces which generalizes the class of uniformly extremely convex Banach spaces is introduced, and some characterizations of these spaces are given. Another paper by N. Khan treats the convergence of new type of double sequences, here introduced, in $n$-normed spaces. An interesting abstract approach to the theory of filter convergence is given in the article by A. Boccuto and $\mathrm{X}$. Dimitriou, in which the links with function spaces and approximation theory are also dealt with. Other aspects of the theory of function spaces and their interconnections with calculus of variations, numerical analysis, complex variables, and stochastic processes are discussed, respectively, in the articles by T. Ma and Y. Feng, H. Wang et al., S. Wang and T. Zhan, and finally P. Duan. These four papers point out how certain methods of general approximation theory in function spaces can be employed in order to solve problems coming from a large variety of mathematical fields. We think that these contributions may represent starting points for new researches in the field of function spaces and approximation theory.

\section{Acknowledgments}

The guest editors wish to express their deep gratitude to all the contributors for the interest showed for our special issue and their interesting articles.

Carlo Bardaro Ioan Rasa

Rudolf L. Stens Gianluca Vinti 


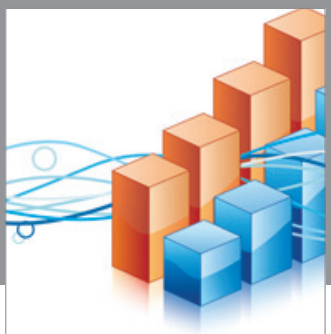

Advances in

Operations Research

vatem alat4

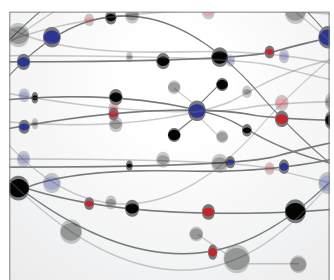

\section{The Scientific} World Journal
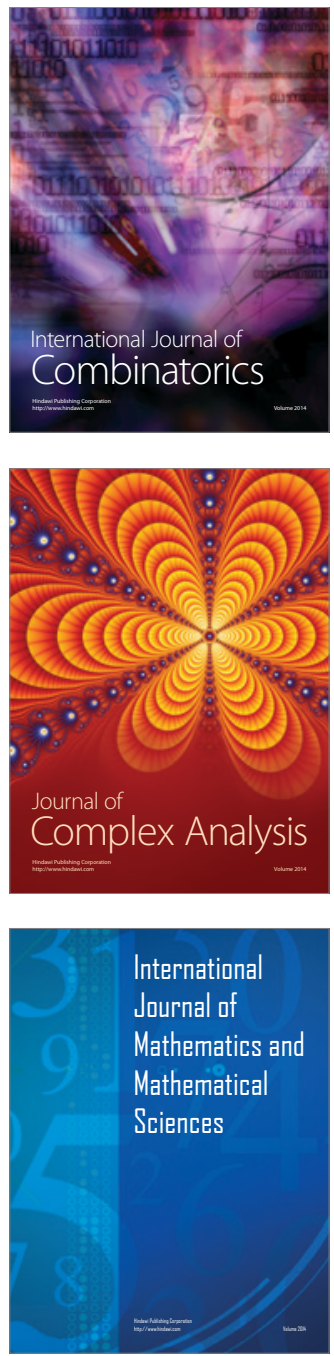
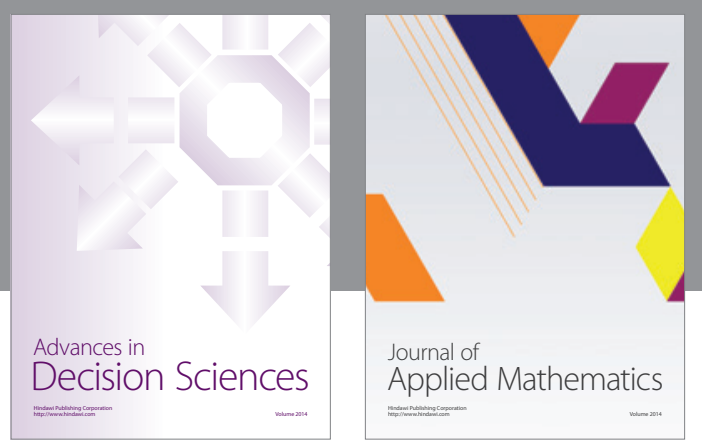

Algebra

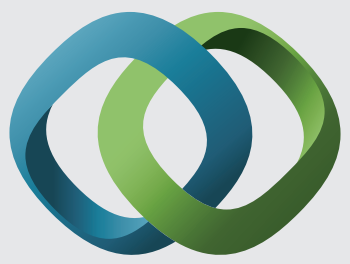

\section{Hindawi}

Submit your manuscripts at

http://www.hindawi.com
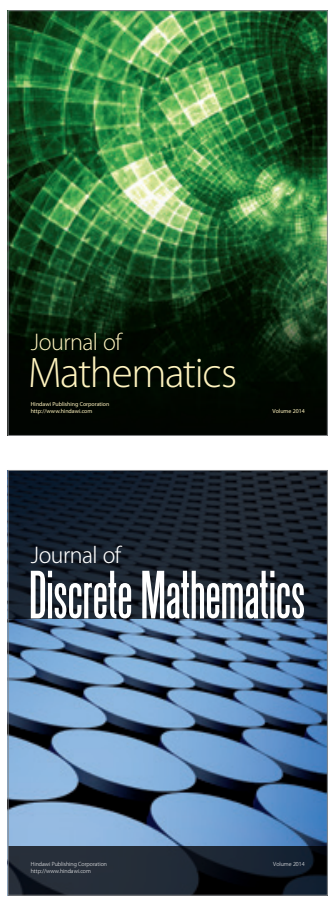

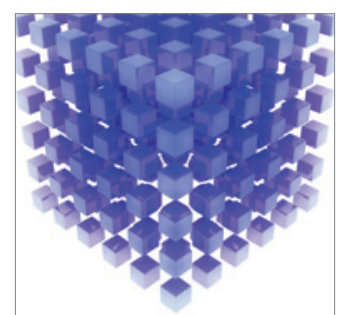

Mathematical Problems in Engineering
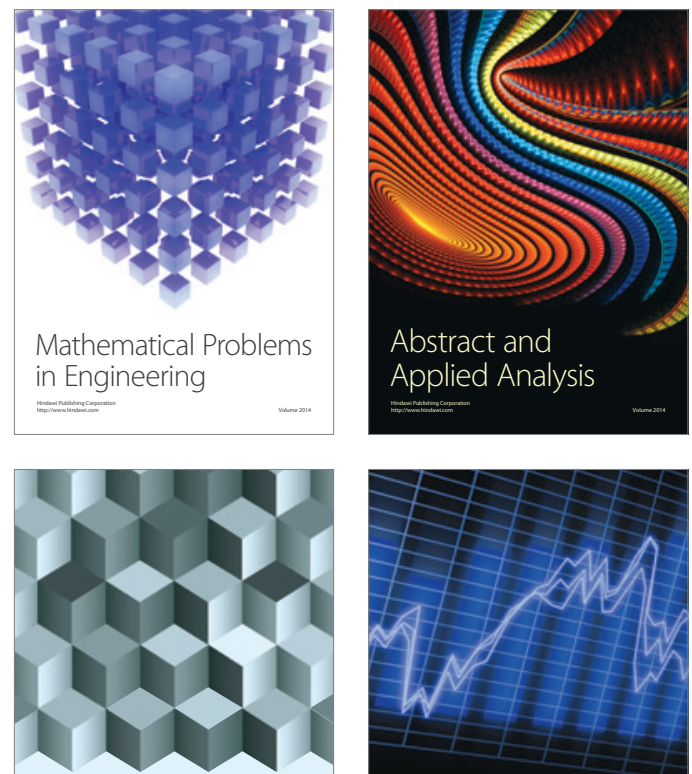

Journal of

Function Spaces

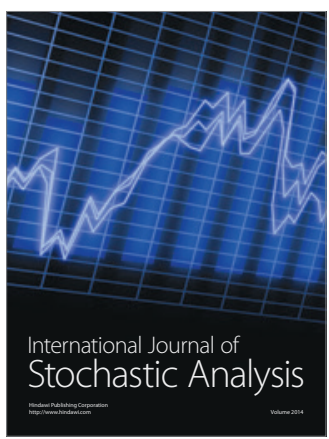

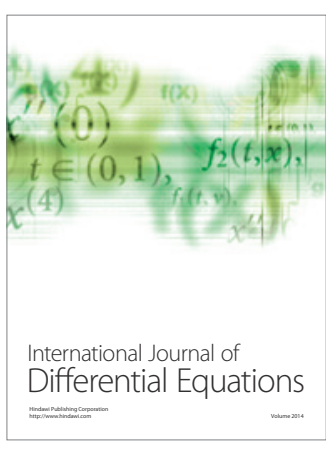
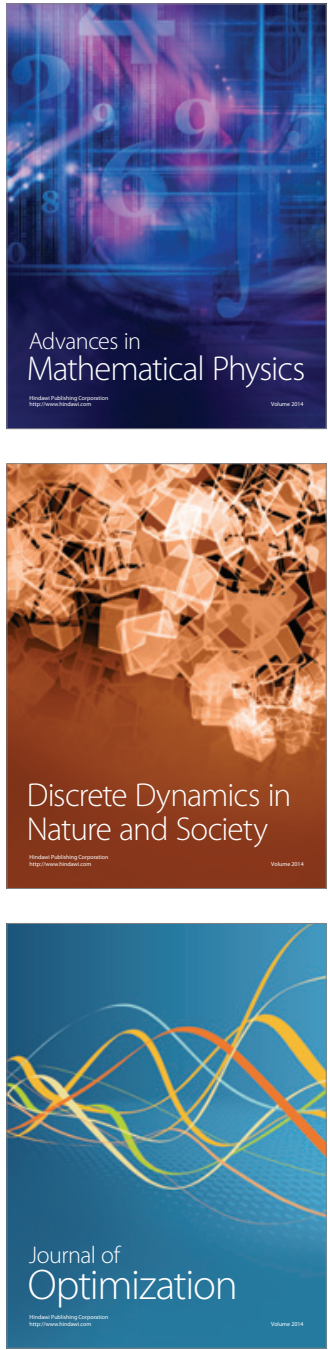